\title{
Notsectio und interdisziplinäre Notfallkonzepte im Kreißsaal „Und wenn es schnell gehen soll?"
}

Lutz Kaufner • Katharina Weizsäcker • Claudia Spies • Aarne Feldheiser • Christian von Heymann

Der geburtsmedizinische Notfall ist durch eine akut lebensbedrohliche Situation für Mutter und/oder Kind charakterisiert. Interdisziplinäre Notfallkonzepte zu Kommunikation, Logistik und Therapie unter Berücksichtigung der strukturellen Voraussetzungen und gesetzlichen Vorgaben der eigenen Klinik können hier helfen, die Handlungsabläufe zu optimieren. Der vorliegende Artikel nennt die wichtigsten geburtsmedizinischen Komplikationen und stellt die interdisziplinären Notfallalgorithmen für 3 der „gefürchtetsten“" geburtsmedizinischen Notfälle vor: Notsectio, peripartale Blutung und Schulterdystokie.

\section{Der geburtsmedizinische Notfall $\nabla$}

Mütterliche Sterblichkeit Der aktuellen Statistik der Weltgesundheitsorganisation (WHO) zufolge geht die mütterliche Sterblichkeit weltweit deutlich zurück (minus 34\% von 1990-2008) [1]. Für die Industrieländer jedoch lässt sich tendenziell ein Anstieg der Mortalität beobachten (plus $16 \%$ von 1990-2008) [2]. In der Auswertung der WHO-Daten wird daher darauf hingewiesen, dass durch eine Optimierung der Therapie geburtsmedizinischer Notfälle die mütterliche Sterblichkeit auch in den Industrienationen reduziert werden könnte [2].

Klassifikation Der geburtsmedizinische Notfall lässt sich nach verschiedenen Ursachen klassifizieren:

- fetale Bradykardie: umfasst alle kindlichen Ursachen, die zu einer geburtsmedizinischen Notfallsituation führen

- Blutung: peripartale Hämorrhagie (z.B. bei Atonie, Uterusruptur)

- Embolie: z.B. Fruchtwasserembolie, Lungenarterienembolie

- Dystokie: z.B. Schulterdystokie, stellvertretend für unerwartete unter der Geburt auftretende Komplikationen

- Hypertonus: z. B. Eklampsie, stellvertretend für hypertensive Notfallsituationen
Standardisiertes Notfallkonzept In vielen der genannten Notfälle muss das Kind mittels Notsectio geboren und neonatologisch versorgt werden und/oder es ist eine intensivierte Notfalltherapie der Mutter erforderlich.

- Damit ist die Notsectio ein zentraler Bestandteil interdisziplinärer Notfallkonzepte für den Kreißsaal.

Die Entwicklung eines standardisierten interdisziplinären Ablaufs für die Notsectio unter Berücksichtigung aller beteiligten Fachdisziplinen und der strukturellen Voraussetzungen der Klinik bildet daher auch die Grundlage für entsprechende interdisziplinäre Behandlungsalgorithmen für die anderen geburtsmedizinischen Notfälle.

\section{Die Notsectio ist ein zentraler Bestandteil inter- disziplinärer Notfallkonzepte für den Kreißsaal.}

\section{Interdisziplinäres Handeln - im Notfall keine leichte Aufgabe}

Interdisziplinarität Interdisziplinäres Handeln im Notfall ist geprägt durch eine Vielzahl verschiedener Schnittstellen, Berufsgruppen und Kommunikationswege sowie unterschiedlicher räumlicher und struktureller Gegebenheiten, gesetzlicher Vorgaben und forensischer Aspekte - und nicht zuletzt durch eine hohe emotionale Belastung für alle Beteiligten.

Gesetzliche Vorgaben Basierend auf dem vom gemeinsamen Bundesausschuss verabschiedeten Stufenmodell der neonatologischen Versorgung [3] wurden Empfehlungen für die strukturellen Voraussetzungen der perinatologischen Versorgung in Deutschland herausgegeben. Daran beteiligt waren die Fachgesellschaften für Gynäkologie und Geburtsmedizin (DGGG), für Anästhesiologie und Intensivmedizin (DGAI), für Neonatologie und pädiatrische Intensivmedizin (GNPI), für Kinderheilkunde und Jugendmedizin (DGKJ) und für Kinderchirurgie (DGKCH). 


\begin{tabular}{|c|c|}
\hline \multicolumn{2}{|c|}{ Regelung der Zuständigkeiten innerhalb der Kommunikation bei einer Notsectio } \\
\hline Indikation & $\begin{array}{l}\text { geburtsmedizinischer Assistenzarzt / Oberarzt: Indikationsstellung } \\
\text { Hebamme: Alarmauslösung, Weisung der eintreffenden Teammitglieder } \\
\text { (automatisierter Rundruf nach festgelegter Rufnummernliste, Gespräche } \\
\text { werden automatisch unterbrochen, spezieller Klingelton) }\end{array}$ \\
\hline Informationsfluss & $\begin{array}{l}\text { Geburtsmediziner: Information an die eintreffenden Teammitglieder } \\
\text { (Mehrlingsgeburt, Indikation, OP- bzw. anästhesierelevante Informationen) }\end{array}$ \\
\hline $\begin{array}{l}\text { gewaschen / } \\
\text { ungewaschen“ }\end{array}$ & $\begin{array}{l}\text { „ungewaschene“ Notsectio (Info durch Geburtsmediziner): } \\
\text { sofortige Narkoseeinleitung } \\
\text { „gewaschene“ Notsectio (Einwaschen der Operateure): } \\
\text { Narkoseeinleitung erst nach Lagerung und Hautdesinfektion wenn OP-Team } \\
\text { schnittbereit am OP-Tisch steht }\end{array}$ \\
\hline Patientenbetreuung & $\begin{array}{l}\text { Anästhesist: Kommunikation mit der Patientin (inkl. Kurzanamnese) } \\
\text { Information an Geburtsmediziner zu Ablaufänderungen mit Angabe von } \\
\text { Grund, zum geplantem Vorgehen und zur erwarteten Dauer bis OP-Freigabe }\end{array}$ \\
\hline
\end{tabular}

\section{Schnittstellen im geburtsmedizinischen Notfall}

\begin{tabular}{|c|c|c|}
\hline $\begin{array}{l}\text { horizontale Schnittstellen } \\
\text { (verschied. Fachabteilungen) }\end{array}$ & $\begin{array}{l}\text { vertikale Schnittstellen } \\
\text { (unterschiedl. Personal) }\end{array}$ & räumliche Schnittstellen \\
\hline $\begin{aligned} & \text { Geburtsmedizin } \\
& \text { (OA, Assistenzarzt, Pflege) } \\
& \text { Anästhesiologie } \\
& \text { (OA, Assistenzarzt, OP-Pflege) } \\
& \text { Neonatologie } \\
& \text { (OA, Assistenzarzt) } \\
& \text { - Radiologie } \\
& \text { Laboratoriumsmedizin } \\
& \text { Transfusionsmedizin }\end{aligned}$ & $\begin{array}{l}\text { ärztlich } \\
\text { (OA, Assistenzarzt) } \\
\text { ärztlich und nicht ärztlich } \\
\text { (Hebammen, Anästhesie- } \\
\text { und OP-Pflege) }\end{array}$ & $\begin{array}{l}\text { Kreißsaal } \\
\text { Sectio-OP } \\
\text { Aufwachraum } \\
\text { Prä-/Postpartalstation } \\
\text { ggf. Intensivstation } \\
\Rightarrow \text { ggf. Angiografie }\end{array}$ \\
\hline
\end{tabular}

Tab. 1

Tab. 2 OA= Oberarzt.
Empfehlungen Die Empfehlungen der DGGG zu den strukturellen Voraussetzungen der perinatologischen Versorgung berücksichtigen u.a. die geburtsmedizinische und anästhesiologische Behandlung von Mutter und Kind und definieren die Anforderungen an die infrastrukturellen Voraussetzungen der Klinik und an die klinikinternen Prozessabläufe [4]. Beispielsweise nennen sie für die Zeit zwischen Indikationsstellung und Kaiserschnitt-Entbindung (Entschluss-Entbindungs-Zeit, E-E-Zeit) bei einer Notlage [5] für ein Perinatalzentrum Level 1 eine E-E-Zeit von $10 \mathrm{~min}+5 \mathrm{~min}$ Wegezeit. Insbesondere diese Empfehlungen zur E-E-Zeit erfordern,

- den Zeitbedarf der eigenen Klinik in Abhängigkeit der Versorgungsstufe für die Notsectio zu ermitteln,

- mögliche Optimierungspotenziale zu identifizieren und

- eine Verbesserung der Prozessabläufe im Rahmen eines interdisziplinären Notfallkonzepts zu erzielen [6].

Die Notsectio ist der häufigste geburtsmedizinische Notfall. Ein entsprechendes Notfallkonzept muss sich nach den Vorgaben zur E-E-Zeit in der eigenen Klinik richten.
Schwachstelle Kommunikation Aufgrund der vitalen Bedrohung von Mutter und Kind sind an der Behandlung geburtsmedizinischer Notfälle eine Vielzahl ärztlicher und nicht ärztlicher Mitarbeiter unterschiedlicher Fachdisziplinen beteiligt. Neben der enormen emotionalen Belastung führt dies zu einer hohen Umgebungslautstärke, einem hohen Stressniveau der Akteure und einer Informationsüberlastung mit einem möglichen Verlust wesentlicher Informationen.

Somit sollten interdisziplinäre Notfallkonzepte wie z. B. für die Notsectio ( $\bullet$ Tab. 1) Regeln für die Kommunikation beinhalten:

- Wer gibt welche Information weiter? Wer führt das Team? (z.B. geburtsmedizinischer und anästhesiologischer Oberarzt)

- einmalige kurze Information an alle Teammitglieder (Lagemeldung)

- kurze adressierte Handlungsanweisungen mit Bestätigung durch ausführende Person (keine generellen Handlungsanweisungen)

-1-zu-1-Kommunikation mit Patientin (z.B. durch Anästhesist)

- Definition der Kommandos (allen Teammitgliedern bekannt): z.B. „gewaschen/ungewaschen“, „Mehrlingsschwangerschaft“ 
Abb. 1

Abb. 2 modifiziert nach [6]. Geburtsmedizin:

Operateur I (OP I): Oberarzt; Operateur II (OP II): Assistenzarzt; verantwortliche Hebamme I (HB I); Hebamme II (HB II) OP-Funktionsdienst: Instrumentierender (IN); Springer (SP) Anästhesie: Anästhesist (AN); Anästhesie-Pflege (AN-P)

Neonatologie: Neonatologe (NEO); Neonatologie-Pflege (NEO-P).

$\mathrm{KS}=$ Kreißsaal;

Rea-Raum =

Reanimationsraum;

US=Ultraschall;

CTG = Kardiotokografie;

AWR = Aufwachraum.

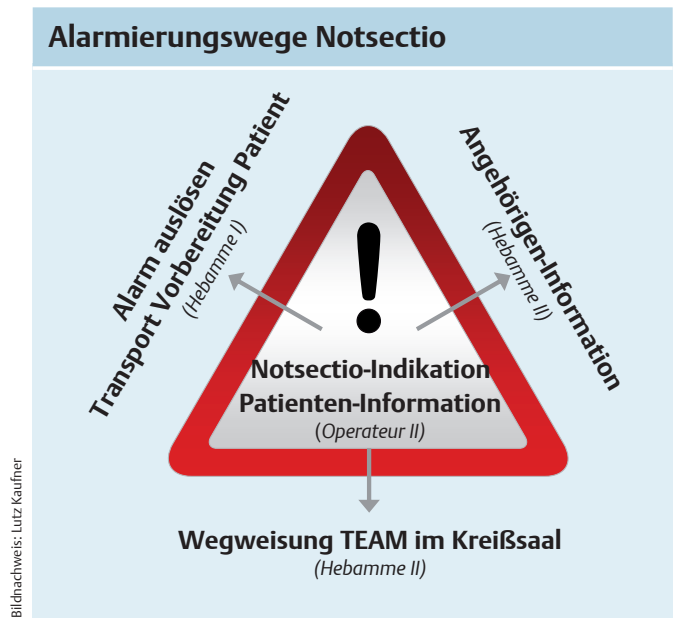

Cave: Kommunizieren nicht vergessen!

Schnittstellenstörungen In der Behandlung geburtsmedizinischer Notfälle ergeben sich durch die Vielzahl der beteiligten Fachdisziplinen und Berufsgruppen verschiedene Schnittstellen ( Tab. 2). An diesen kann es insbesondere in Notfallsituationen zu Störungen kommen:

- z.B. unvollständige geburtsmedizinische Kurzanamnese an die anderen Fachdisziplinen, unklare Kommandos

> z.B. Hebammen-OP-Pflege: falsche Priorisierung von Einzelhandlungen (z.B. Fixierung der
Patientin noch vor Anlage des Blasenkatheters durch die Hebamme bei Notsectio)

z.B. Transport der Patientin aus dem Kreißsaalzimmer in OP, Übergabe des Kindes in die neonatologische Versorgung

\section{Notsectio}

Häufige Notfallsituation Die Notsectio ist eine der häufigsten geburtsmedizinischen Notfallsituationen. Nicht nur in Zentren (Level 1) mit erhöhtem Anteil an Frühgeburten oder Risikoschwangerschaften, sondern auch in Kliniken mit geringeren Geburtenzahlen sollte ein entsprechend an die strukturellen Voraussetzungen angepasstes interdisziplinäres Handlungskonzept hinterlegt sein und regelmäßig trainiert werden [6].

Konzeptentwicklung Für die Konzeptentwicklung müssen zunächst in einer Bedarfsanalyse (z.B. im Rahmen einer interdisziplinären Notsectio-Übung) mögliche Mängel im Ablauf unter Berücksichtigung der Vorgaben an die E-E-Zeiten identifiziert werden. Danach richten sich:

- interdisziplinäre Vereinbarung zur Kommunikation (๑ Tab. 1)

- Alarmierungsweg, Zuständigkeit Patiententransport

- Priorisierung und direkte Zuordnung der Einzelhandlungen jedes Akteurs

\section{Priorisierung der Einzelhandlungen aller Akteure bei einer Notsectio im Sectio-OP}
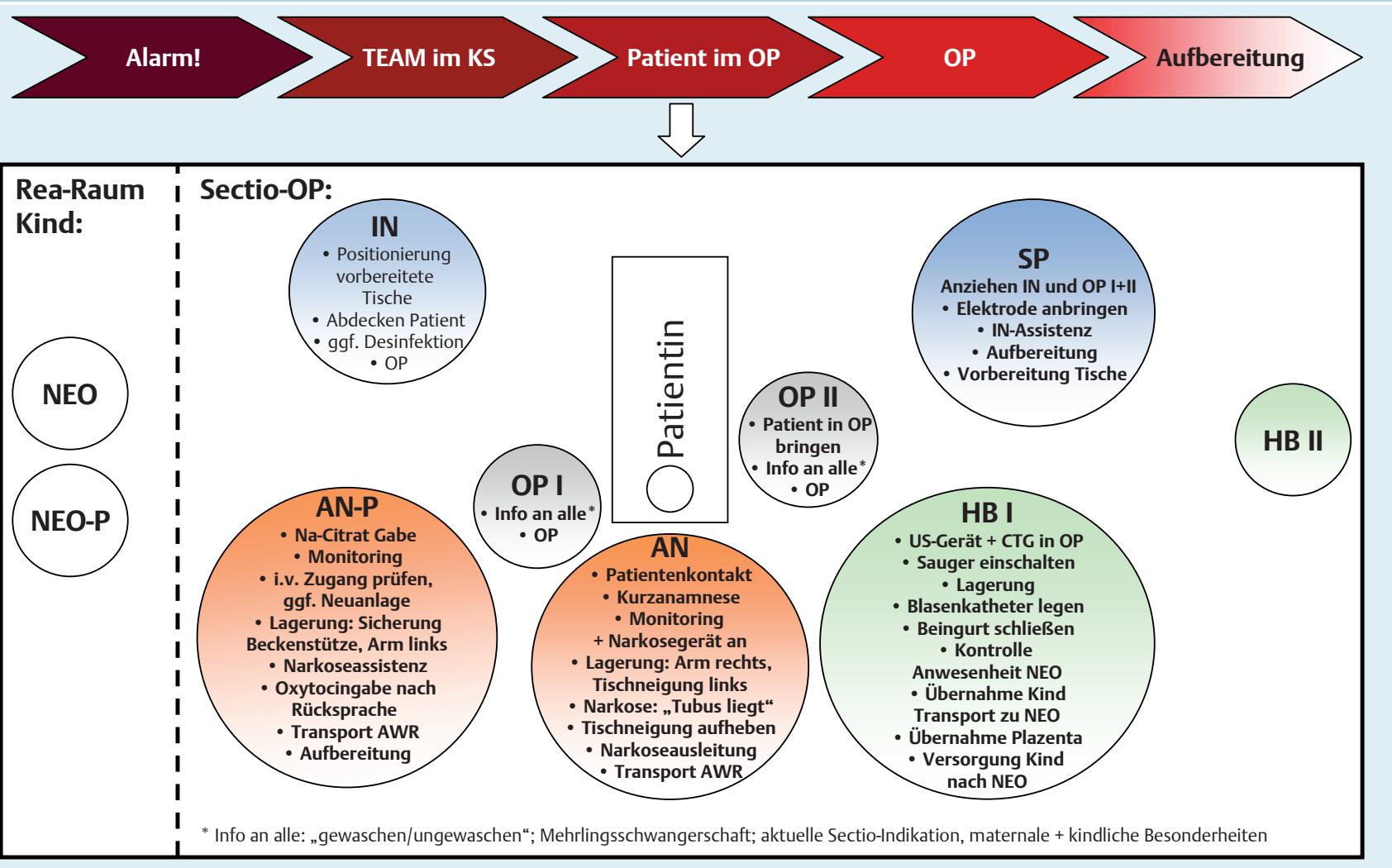
- Teamzusammenstellung:

$\triangleright$ Geburtsmedizin:

Operateur I: Oberarzt; Operateur II: Assistenzarzt; verantwortliche Hebamme I; Hebamme II

$\triangleright$ OP-Funktionsdienst: Instrumentierender; Springer

$\triangleright$ Anästhesie: Anästhesist; Anästhesie-Pflege

$\triangleright$ Neonatologie: Neonatologe; NeonatologiePflege

Alarmierung Der Notsectio-Alarm kann z. B. als Rundruf mit speziellem Klingelton nach festgelegter Rufnummernliste (Teamzusammenstellung) über eine Kurzwahltaste am zentralen Telefon im Kreißsaal ausgelöst werden. Laufende Telefonate werden unterbrochen und alle Teammitglieder finden sich unverzüglich im Sectio-OP ein. Mit der Alarmierung geht der Transport der Patientin in den Sectio-OP einher.

- Der Alarmierungsweg und die entsprechenden Zuständigkeiten müssen vorab definiert sein (o Abb. 1).

Priorisierung der Einzelhandlungen Jedem Teammitglied müssen entsprechend seiner Berufsgruppenzugehörigkeit, fachlichen Kompetenz und rechtlichen Verantwortung seine Einzelhandlungen priorisiert eindeutig zugeordnet $\operatorname{sein}(\odot$ Abb. 2).

- Dies soll den Ablauf trainierbar machen und Zeitverluste durch wiederholte oder fehlende Handlungen vermeiden (z.B. die orale $\mathrm{Na}^{+}-$ Citrat-Gabe durch Anästhesist und/oder Anästhesiepflegekraft).

Durch die Priorisierung und Strukturierung der Einzelhandlungen können sich die einzelnen Teammitgliedern auf Ihre Aufgaben konzentrieren. Über diese Harmonisierung der Abläufe kann in einem zweiten Schritt auch die E-E-Zeit reduziert werden.

Training In einer clusterrandomisierten Untersuchung mit und ohne Teamtraining konnte eine Verkürzung der E-E-Zeit für die Interventionsgruppe erzielt werden [7].

- Dabei ist ein simulationsbasiertes Training einem didaktischen Training im Hinblick auf den Lernerfolg und auf die Fehlervermeidung überlegen [8].

Dies setzt ein Simulationstraining an Originalschauplätzen und in einem festen zeitlichen Turnus (z. B. monatlich) voraus. Bei diesem trainieren alle beteiligten Berufsgruppen und Teammitglieder den Ablauf von der Indikationsstellung im Kreißsaalzimmer über den Transport in den OP bis hin zur „Abnabelung des Kindes“.

Fester Bestandteil klinischer Routine Da die Notsectio häufig Bestandteil der Notfalltherapie geburtsmedizinischer Komplikationen ist, sollte das Notsectio-Training obligat interdisziplinär

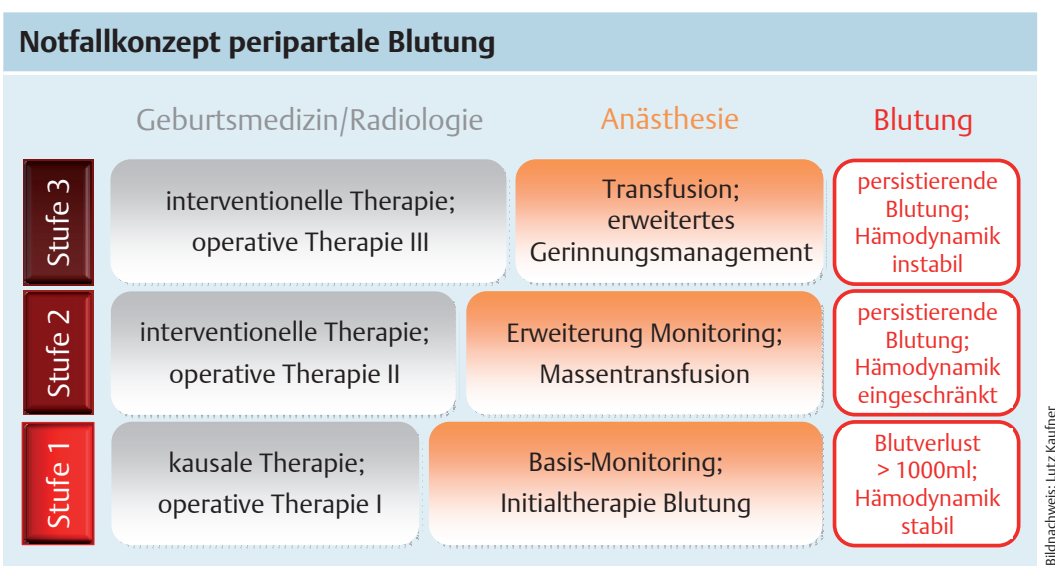

durchgeführt und für alle Beteiligten zu einem Bestandteil ihrer klinischen Routine werden.

Praxistipp Notsectio-Training: 2 Simulationsdurchläufe pro Trainingstermin mit einer Gesamtzeit von ca. 45 min: E-E-Simulationszeit < $15 \mathrm{~min}$. Die Übungsfrequenz ist an die klinikinternen Rotationen neuer Weiterbildungsassistenten anzupassen.

\section{Peripartale Blutung}

Führende Ursache mütterlicher Mortalität Die postpartale Hämorrhagie ist weiterhin eine der führenden Ursachen mütterlicher Mortalität $[2$, 9]. Bedingt durch ihre Dynamik, die schwangerschaftsbedingten Änderungen der Blutgerinnung und die Vielzahl verzahnter Therapieansätze [10] erfordert sie ein besonderes interdisziplinär abgestimmtes Management (inkl. Labor- und Transfusionsmedizin, interventionelle Radiologie).

Konzeptentwickung Schwerpunkt eines interdisziplinären Notfallkonzepts ist die Integration aller Therapieoptionen in ein Eskalationsschema ( $\odot$ Abb. 3) unter Berücksichtigung der strukturellen Voraussetzungen der Klinik (z.B. Zeiten bis zum Erhalt von Blutprodukten). Im Gegensatz zur Notsectio, bei der die Handlungen der einzelnen Teammitglieder genau priorisiert sind und fast „automatisch“ ablaufen, müssen sich Operateur und Anästhesist bei der postpartalen Hämorrhagie aufgrund der Vielzahl der Blutungsursachen und Behandlungsoptionen immer wieder erneut kurz abstimmen.

Dennoch sollten die einzelnen Handlungen in einem Eskalations- bzw. Stufenschema vorab interdisziplinär definiert werden. So wird gewährleistet, dass jeder im Team weiß, welche Therapie von der jeweils anderen Fachdisziplin zeitgleich durchgeführt wird und welche Therapieoption sich anschließt.
Abb. 3 Stufenschema für die geburtsmedizinische, ggf. radiologische und anästhesiologische Behandlung der peripartalen Blutung in Abhängigkeit von Blutverlust und Hämodynamik. 


\section{Therapie der Blutung}

Stufe 1

(Blutverlust > $1000 \mathrm{ml}$, Hämodynamik stabil)

\section{Stufe 2}

(persistierende Blutung, Hämodynamik eingeschränkt)

(persistierende Blutung, Hämodynamik instabil)

\section{Stufe 3}

\section{Anästhesiologie:}

Anlage großvolumiger Venenverweilkanüle, adäquate Volumentherapie (z. B. kolloidale Lösungen), Blutgasanalyse, Entnahme Ausgangslabor (BB, Quick, PTT, AT, FBG, D-Dimere);

Anforderung von Notfallblutprodukten, ggf. Gabe von lyophilisiertem FFP, uterotone Medikation (ggf. Stopp Oxytocininfusion, ggf. Misoprostolgabe sowie Sulprostoninfusion);

Tranexamsäure (1-2g als Bolus i.v.)

\section{Geburtsmedizin:}

Uteruskompression, -massage, Ultraschallkontrolle, ggf. manuelle Nachtastung bei Plazentaretention, ggf. lokale Blutstillung bei Verletzungen der Geburtswege

\section{- Anästhesiologie:}

Erweiterung des Monitoring (Arterie, ggf. ZVK); zusätzliches Personal; Bereitstellung Massentransfusionsgerät; ggf. Intubation;

Beginn von Transfusion, Volumen- und Katecholamintherapie; Wärmemanagement, ggf. Bereitstellung Cell-Saver

\section{- Geburtsmedizin/Radiologie:}

operative Blutstillung (z. B. Uteruskompressionsnähte); ggf. Bakri-Katheteranlage; ggf. Verschluss der uterinen Gefäßversorgung (interventionelle Radiologie oder Clipping bzw. Ligatur der Aa. uterinae)

\section{- Anästhesiologie:}

Tranexamsäure (6-10 mg/kg KG/h); FFP und EK (Verhältnis 2:1); 2-4g Fibrinogen; PPSB (Bolus 20-25 iE/kg KG);

2. Laborentnahme, ggf. Thrombelastogramm, BGA, ggf. TK notwendig; $\mathrm{Ca}^{2+}$-Substitution ggf. FXIII (15-20 iE/kg KG nach Spiegelbestimmung)

\section{- Geburtsmedizin/Radiologie:}

Fortführung der Therapie aus Stufe 2, ggf. Hysterektomie

Tab. 3 Therapie der Blutung gemäß der Leitlinienempfehlung der Deutschen Gesellschaft für Gynäkologie und Geburtshilfe [12]. $\mathrm{BB}=$ Blutbild;

PTT = partielle Thromboplastinzeit;

AT = Antithrombin;

FBG = Fibrinogen;

$\mathrm{FFP}=$ gefrorenes Frischplasma; ZVK= zentraler Venenkatheter; EK= Erythrozytenkonzentrat; PPSB = Prothrombinkonzentrat; $\mathrm{BGA}=$ Blutgasanalyse; $\mathrm{TK}=$ Thrombozytenkonzentrat; FXIII = Faktor XIII.

\section{Cave Peripartale Blutungen verlaufen häufig äußerst fulminant! Die Rekrutierung zusätzlichen Personals sollte priorisierter Bestandteil eines interdisziplinären Notfallkonzepts sein.}

Eskalationsschema In Abhängigkeit von der Schwere des Blutverlusts (WHO-Klassifikation) [11] und der hämodynamischen Beeinträchtigung lassen sich 3 Stufen unterscheiden. Diesen sind alle möglichen Handlungsoptionen zugeordnet. Dabei sind die Übergänge zwischen den Stufen fließend, die Handlungen der vorangegangenen Stufe jedoch meist Voraussetzung für die folgende Stufe.

Die Therapie der Blutung richtet sich gemäß der Leitlinienempfehlung der DGGG [12] nach der Höhe des Blutverlusts und muss an die strukturellen Voraussetzungen der jeweiligen Klinik angepasst sein ( $\bullet$ Tab. 3 ).
Gerinnungssituation Neben der erweiterten operativen und interventionell-radiologischen Therapie steht vor allem die Optimierung der Gerinnungssituation im Vordergrund. Zwar kann in der Initialtherapie nicht auf Laborparameter gewartet werden, jedoch dienen die regelmäßigen Laborentnahmen der späteren Kontrolle der Therapiemaßnahmen. Zielparameter sind

> Quick-Wert (>50\%),

- partielle Thromboplastinzeit (PTT<50s),

- Thrombozytenzahl (>50/nl),

- Fibrinogen (FBG $>150 \mathrm{mg} / \mathrm{dl}$ ),

- $\mathrm{Ca}(>0,9 \mathrm{mmol} / \mathrm{l})$,

$>\mathrm{pH}(>7,2)$,

- $\mathrm{Hb}(>8 \mathrm{~g} / \mathrm{dl})$ und

- Temperatur $\left(>35^{\circ} \mathrm{C}\right)$.

Rekombinanter Faktor VIla Die Entscheidung zur Applikation von rekombinantem Faktor VIIa, als Ultima-Ratio-Therapie in der Leitlinie der DGGG empfohlen [12, 13], sollte im interdisziplinären Konsens getroffen werden. Am Ende der Behandlungsstufe 3 bei persistierender Blutung trotz Optimierung der Gerinnungsbedingungen ist dieser Behandlungsschritt vor der Hysterektomie in Erwägung zu ziehen.

\section{Neben der operativen und interventionell-radiolo- gischen Therapie steht vor allem die Optimierung der Blutgerinnungssituation im Vordergrund.}

Logistik Die Anforderungen an die Logistik zur Behandlung der peripartalen Blutung sind erheblich. Dabei sollten vorab interdisziplinär folgende Punkte geklärt werden:

- Wo ist das nächstes Depot für Plasmaderivate? Wer geht hin (z. B. Hebamme)? Was wird benötigt (z.B. Patientenetikett)?

- Bereitstellung von lyophilisiertem Frischplasma + Tranexamsäure in einem Kreißsaal-Notfalldepot (Anästhesiepflege)

- Bestellung von Notfallblutprodukten, Information der Blutbank (Anästhesiepflege 1)

- Wird zusätzliches Personal benötigt? ggf. Notruf „Blutung“ (v.a. Anästhesie Arzt + Pflege)

- mobiles Massentransfusionsgerät und CellSaver-Reservoir an definiertem Standort in OPNähe - wer rüstet auf (Anästhesiepflegekraft 2)?

- Information bzw. Alarmierung der Radiologie (z.B. Oberarzt Geburtsmedizin) oder des operativen Hintergrunddienstes

- Breitstellung von Anästhesieequipment im Angiografieraum (Anästhesiepflegekraft 2)

- Organisation der intensivmedizinischen Überwachung, ggf. in OP-Nähe (Geburtsmedizin/ Anästhesiologie)

- Organisation der Labordiagnostik „Bedside“: Thrombelastogramm, ggf. Thrombozytenzahl (z.B. auf Intensivstation, Transportblutentnahme Röhrchen + Info Intensivstation durch Anästhesiepflegekraft 2), BGA (Anästhesiepflegekraft 1) 
Ein kreißsaalnahes Notfalldepot mit lyophilisiertem Frischplasma und Tranexamsäure ermöglicht eine frühzeitige Gerinnungstherapie bis zum Eintreffen der Notfallblutprodukte.

Training Im Gegensatz zur Notsectio lässt sich das interdisziplinäres Management der peripartalen Blutung effektiv nur in einer dynamischen Simulation in Simulationszentren oder mit speziellen Simulationspuppen vor Ort trainieren. Daher ist es umso wichtiger, interdisziplinär einen Algorithmus entsprechend den strukturellen Voraussetzungen der eigenen Klinik zu konsentieren, diesen an definierten Orten (z. B. Sectio-OP, Aufwachraum) gut sichtbar auszuhängen und z. B. im Rahmen des Notsectio-Trainings immer wieder zu üben - vor allem mit neuem Personal.

\section{Schulterdystokie}

Geburtsmedizinischer Notfall außerhalb des OPs Die Schulterdystokie ist ein seltener, aber häufig fulminant verlaufender und überraschender Notfall der vaginalen Geburt. Dabei ist die Geburt des Kindes durch die unvollständige Entwicklung der anterioren Schulter erschwert und häufig durch ein Zurückweichen des Kopfes in den Vulva-Dammbereich gekennzeichnet (Turtle-Phänomen) [14].

- Die Schulterdystokie ist mit einer hohen Rate an neonataler Morbidität, Schädigungen des Plexus brachialis und möglichen Skelettverletzungen des Kindes vergesellschaftet [14].

Große Stressbelastung Neben dem traumatisierenden Erleben für die Mutter unter der Geburt ist auch das behandelnde geburtsmedizinische Team einer großen Stressbelastung ausgesetzt: Es muss binnen kürzester Zeit eine Vielzahl von Manövern zur Lösung der Schulter in Erwägung ziehen und zügig eine Entscheidung treffen im Sinne der Anästhesieeinleitung und ggf. der operativen Therapie. Dabei steht die Schulterdystokie stellvertretend für alle plötzlich unter der vaginalen Geburt auftretenden Notfälle im Kreißsaalzimmer, die aufgrund der räumlichen Bedingungen ein gewisses Maß an Improvisation vom behandelnden Team verlangen.

Konzeptentwicklung Anders als bei der Notsectio und der peripartalen Blutung ist ein detailliertes interdisziplinäres Vorgehen bei der Schulterdystokie weder in der Literatur noch in den Leitlinien hinterlegt. Konkret empfiehlt die Leitlinie der DGGG die Alarmierung des Anästhesisten [14]; genaue Zeitpunkte und anästhesiologische Anforderungen werden nicht genannt.

- Darüber hinaus empfiehlt die Leitlinie, dass jede geburtsmedizinische Klinik über einen Managementplan verfügen und diesen in regelmäßigen Abständen trainieren sollte [14].

\section{„HELPERR“-Schema}

$$
\begin{aligned}
& \underline{H} \text { - Help } \\
& \underline{E} \text { - Episiotomy } \\
& \underline{\underline{L}} \text { - Legs (= McRoberts) } \\
& \underline{\mathrm{P}} \text { - Pressure (= suprasymphysärer Druck) } \\
& \underline{E} \text { - Extract posterior arm } \\
& \underline{\mathrm{R}} \text { - Rotate shoulders internally } \\
& \underline{\mathrm{R}} \text { - Rotate mother (Vierfüßlerstand etc.) }
\end{aligned}
$$

Aus diesem Grund sollte ein gemeinsames interdisziplinäres Notfallkonzept zwischen Geburtsmedizin und Anästhesiologie entsprechend der strukturellen Voraussetzungen der Klinik abgestimmt und trainiert werden. Dabei müssen sowohl dem Geburtsmediziner als auch dem Anästhesisten die konkreten Handlungen zugewiesen und die therapeutischen Maßnahmen des jeweiligen Partners und deren Konsequenzen bewusst sein:

Anästhesiologie Der Anästhesist muss wissen:

- Notfallsituation mit absoluter Gefahr für das kindliche Leben

- welche Manöver in welcher Reihenfolge

- wann Narkoseeinleitung

- wenn Narkoseindikation gestellt: schnellstmögliches sicheres Handeln

- eingeschränkte räumliche Bedingungen

- anästhesierelevante Risikofaktoren der Schulterdystokie: u.a. Adipositas, Diabetes

Geburtsmedizin Der Geburtsmediziner muss wissen:

- keine Alternativen zur Allgemeinanästhesie (z.B. symptomatische Schmerztherapie), d.h. „Alles oder Nichts“-Entscheidung

Einsatz der Allgemeinanästhesie in der Behandlung der Schulterdystokie

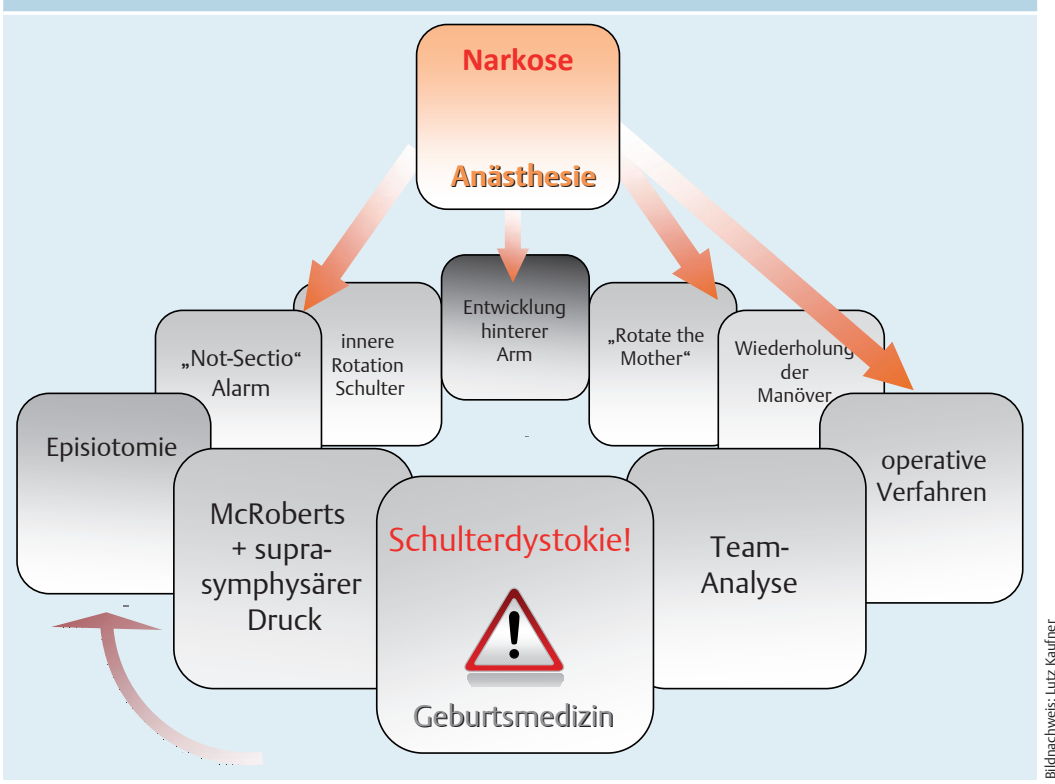

Abb. 4 „HELPERR“- Schema als Hilfestellung der geburtsmedizinischen Therapie zur Behandlung der Schulterdystokie. 


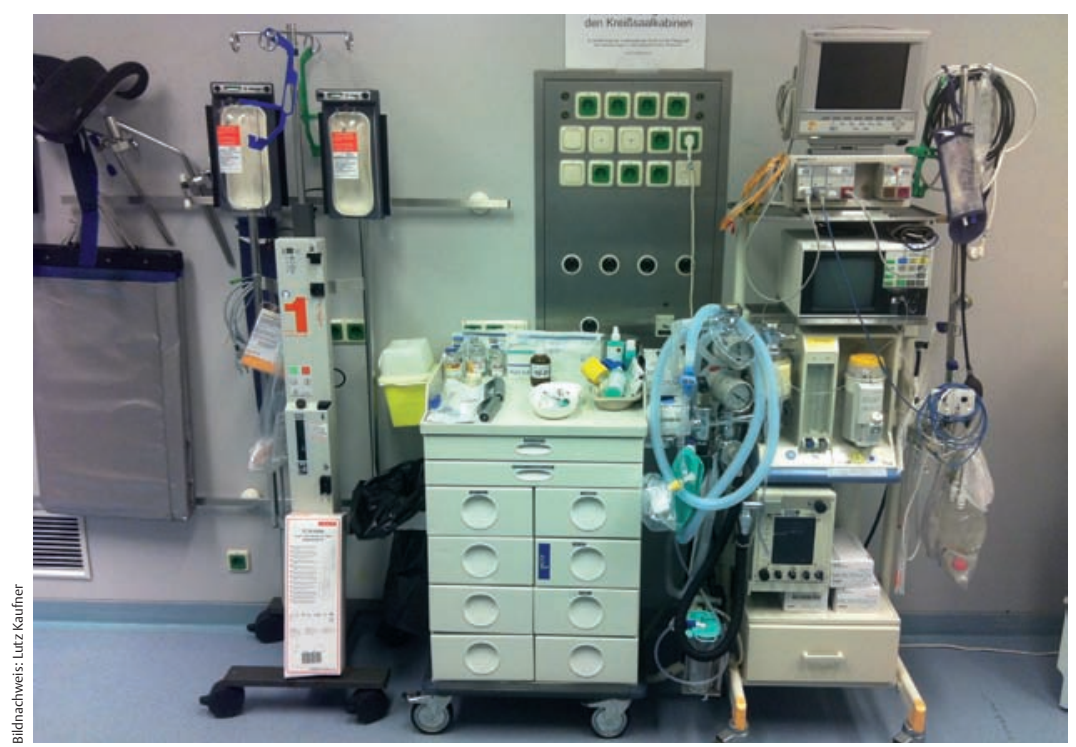

Abb. 6 Um bei der Narkoseeinleitung keine Zeit für den Geräteanschluss und die Narkosevorbereitung zu verlieren, sollten Notfall-Narkosematerialien für den Einsatz im Kreißsaalzimmer bereitstehen. rechts: Narkosegerät (mobiles Flaschengerät);

Mitte: Narkosewagen mit Notfall-Medikamentenschale und Intubationsbesteck; links: mobiles Massentransfusionsgerät.
- trotz schnellen Handelns - Narkoseeinleitung braucht Zeit

- kurze direktive Information an das Anästhesieteam „Narkose jetzt!“ erforderlich

- Muskelrelaxierung ggf. hilfreich, aber nur in Allgemeinanästhesie möglich

Cave Risikofaktoren für Schulterdystokie sind zum Teil auch Risikofaktoren für die Anästhesie.

Therapie In der akuten Therapie der Schulterdystokie gibt das HELPERR-Schema ( Abb. 4) einen Überblick über die wesentlichen geburtsmedizinischen Maßnahmen. Dabei sind

- die Erweiterung der Episiotomie,

- das Beenden einer laufenden Oxytocin-Infusion, - die i.v. Tokolyse und

- die Stellungsänderung der Symphyse (McRoberts-Manöver)

in $40 \%$ der Fälle erfolgreich und meist noch vor Eintreffen des Anästhesieteams abgeschlossen [15].

Zwar gibt es auch in der Literatur keine eindeutigen Untersuchungen zu Bedeutung und Zeitpunkt der Anästhesie und einer möglichen Muskelrelaxierung. Dennoch wird in verschiedenen Behandlungsalgorithmen empfohlen, dass nach erfolglosem McRoberts-Manöver alle weiteren Manöver in Allgemeinanästhesie durchgeführt werden sollten (u.a. innere Rotation der Schulter, Lösung des hinteren Armes bis hin zum abdominellen Rettungsversuch) ( $\odot$ Abb. 5) $[15,16]$.

Narkose außerhalb des OPs Wird vom Geburtsmediziner die sofortige Allgemeinanästhesie zur Behebung der Schulterdystokie gefordert, muss diese unverzüglich, schnell und sicher eingeleitet werden. Dabei wird das Anästhesieteam vor verschiedene Herausforderungen gestellt:

- schnelles Arbeiten - hohes kindliches Risiko für Asphyxie und Kreislaufstillstand
- viele Personen und Geräte am Bett der Patientin

- hohe Umgebungslautstärke, hektische Atmosphäre

- häufig kein maternaler Kontakt im Sinne einer Kurzanamnese mehr möglich

- klares Signal zum Narkosebeginn von Geburtsmediziner einfordern

- Anästhesist muss ans Kopfende der Patientin, Abrücken des Bettes unter laufender geburtsmedizinischer Therapie

- Handlungsfreiraum für Anästhesiepflegekraft sowie Narkosegerät und Anästhesiewagen schaffen

- hohes maternales Aspirationsrisiko

Abstimmung Die Narkoseeinleitung im Kreißsaalzimmer sollte mit der geburtsmedizinischen Therapie abgestimmt und trainiert werden. Hierfür müssen folgende Punkte geklärt sein:

- Standort des Anästhesiewagens (Intubationsbesteck liegt bereit) und des mobilen Narkosegeräts sowie der Notfallmedikamentenschale (z.B. fertig aufgezogen; mit Datum, Namen und Uhrzeit versehen; $\triangle$ Abb. 6)

- Wer nimmt was? (z.B. Anästhesiepflegekraft den Anästhesiewagen; Anästhesist das Narkosegerät)

- Art des Narkosegerätes (batteriebetriebenes Gerät mit Gasflaschenversorgung)

- Einweisung des eintreffenden Anästhesieteams in das Kreißsaalzimmer (z.B. durch die Hebamme)

- Priorisierung der Einzelhandlungen: klares Vorgehen bzgl. Anschluss Monitoring und Narkoseeinleitung

Zeitverluste vemeiden Um die Narkose bei Schulterdystokie unmittelbar einleiten zu können, müssen Zeitverluste für den Geräteanschluss und für die Narkosevorbereitung unbedingt vermieden werden. Aus diesem Grund sollte ein autonomes mobiles Narkosegerät inklusive Absaugung und Kapnometrie verwendet werden, das durch Öffnen der Sauerstoffflasche sofort betriebsbereit ist ( $\triangle$ Abb. 6).

Die bereits fertig aufgezogenen Notfall- und Narkosemedikamente in einer Medikamentenschale sowie das vorbereitete Intubationsbesteck ermöglichen der Anästhesiepflegekraft, sich um die Narkosevorbereitung der Patientin zu kümmern (Lagerung der Patientin, Prüfen des Venenzugangs, Anschluss Infusion, Anschluss Sauerstoffsättigung).

Zeitverluste für den Geräteanschluss und die Narkoseeinleitung sind unbedingt zu vermeiden.

Narkoseeinleitung Sobald die unbedingt notwendigen Anästhesievorbereitungen abgeschlossen sind, wird unmittelbar mit der Präoxygenierung begonnen und mittels „Rapid Sequence 
Induction“ (RSI) die Narkose eingeleitet und dem behandelnden Geburtsmediziner der Narkosestatus übermittelt. Ist der Atemweg gesichert, können die erweiterten Maßnahmen zur Narkoseführung eingeleitet werden (RR-Messung, EKGMonitoring; Strom- und Gasanschluss des Narkosegeräts etc.).

- In Rücksprache mit dem Geburtsmediziner sollte eine ausreichende Muskelrelaxierung der Patientin gewährleistet sein.

Aufgrund des fulminant traumatischen Ereignisses für Mutter, Angehörige und das behandelnde Team ist es ratsam, neben einer intensivierten Betreuung der Patientin auch innerhalb des behandelnden Teams die Notfallsituation interdisziplinär auszuwerten (z.B. als Perinatalkonferenz mit Neonatologen).

Fazit Im geburtsmedizinischen Notfall ist zügiges Handeln essenziell für das kindliche und/oder mütterliche Überleben. Interdisziplinär trainierbare Notfallkonzepte können helfen, Abläufe zu optimieren, Zeitverluste zu vermeiden und dem Team die notwendige Sicherheit in der Notfalltherapie zu geben. 4

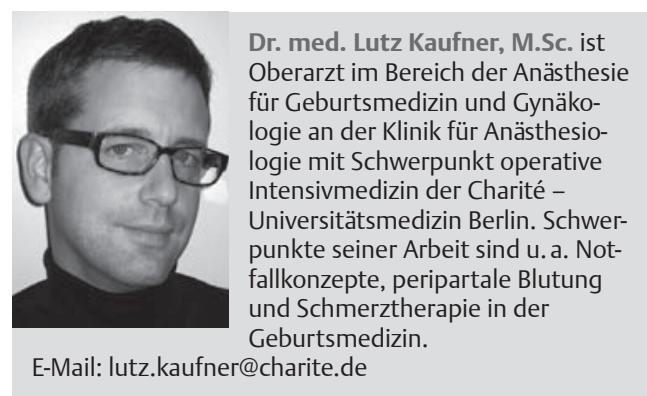

Dr. med. Katharina Weizsäcker ist Leitende Oberärztin an der Klinik für Geburtsmedizin der Charité Universitätsmedizin Berlin, Campus Virchow-Klinikum. Schwerpunkte ihrer Arbeit sind u. a. Infektionen in der Schwangerschaft und Kreißsaalmanagement. E-Mail: katharina.weizsaecker@charite.de

Prof. Dr. med. Claudia Spies ist seit 2005 Direktorin der Klinik für Anästhesiologie mit Schwerpunkt operative Intensivmedizin, Charité - Universitätsmedizin Berlin, Campus Virchow-Klinikum und Campus Mitte. E-Mail: claudia.spies@charite.de

Dr. med. Aarne Feldheiser ist Oberarzt an der Klinik für Anästhesiologie mit Schwerpunkt operative Intensivmedizin, Charité - Universitätsmedizin Berlin, Campus Virchow-Klinikum. Schwerpunkte seiner Arbeit sind die Anästhesie und Hämodynamikforschung im Bereich der Geburtsmedizin und Gynäkologie.

E-Mail: aarne.feldheiser@charite.de

Prof. Dr. med. Christian von Heymann, DEAA ist stellvertretender Direktor der Klinik für Anästhesiologie mit Schwerpunkt operative Intensivmedizin, Charité - Universitätsmedizin Berlin, Campus Charité Mitte und Campus Virchow-Klinikum.

E-Mail: christian.von_heymann@charite.de

\section{Kernaussagen}

Auch in den Industrienationen ist die schwere peripartale Hämorrhagie eine der führenden Ursachen mütterlicher Sterblichkeit.

Die Notsectio ist häufig zentraler Bestandteil der Therapie verschiedener geburtsmedizinischer Komplikationen.

Ein interdisziplinäres Notfallkonzept zur Notsectio und ein regelmäßiges Training können helfen, die interdisziplinären Handlungen innerhalb der Notsectio zu harmonisieren. Dies ist insbesondere dann wichtig, wenn zusätzlich begleitende maternale Komplikationen behandelt werden müssen (z. B. atone Blutung).

In einem Notfallkonzept zur Notsectio müssen die Teamzusammensetzung, die Alarmierung, die Kommunikation, die Priorisierung der Einzelhandlungen, die Auswertung und das Training interdisziplinär abgestimmt werden.

Jedes Teammitglied muss im Notfall wissen, welche Therapie von der jeweils anderen Fachdisziplin zeitgleich durchgeführt wird und welche Therapieoptionen sich anschließen.

Zur Therapie der schweren peripartalen Blutung gehören verschiedene operative, aber auch interventionell-radiologische Therapieansätze. Sie geht einher mit einer stetigen Optimierung der Blutgerinnungssituation.

Ein kreißsaalnahes Notfalldepot mit lyophilisiertem Frischplasma und Tranexamsäure ermöglicht eine frühzeitige Gerinnungstherapie bis zum Eintreffen der Notfallblutprodukte.

Das hohe kindliche Asphyxierisiko bei der Schulterdystokie erfordert ein schnelles Handeln aller beteiligten Teammitglieder.

Für die Narkoseeinleitung bei einer Schulterdystokie müssen Zeitverluste für Geräteanschluss und Narkosevorbereitung unbedingt vermieden werden.

Ein regelmäßiges interdisziplinäres Simulationstraining hinterlegter Notfallkonzepte an Originalschauplätzen kann helfen, Prozessabläufe für den Notfall zu optimieren.

Interessenkonflikt Claudia Spies hat für den vorliegenden Beitrag nicht relevante Fördergelder erhalten von Abbott, Aspect, Baxter, Care Fusion, Deltex Medical, Dräger, Fresenius, Hutchinson, Köhler, MSD, MCN, Novartis, Roche, Sysmex, University Hospital Stavanger. Ihre Klinik hat öffentliche Fördergelder bekommen von Argus, BDA, BMBF, DKH, DLR, German Research Society, GIZ, Universitätsmedizin Berlin, Stifterverband. Außerdem hat Claudia Spies Vortragshonorare von Abbott, B.Braun, Baxter, Essex Pharma, GSK und Bispebjerg Hospital sowie Reisekostenunterstützung von Abbott, Aspect, Baxter, Fresenius und Wyeth erhalten.

Aarne Feldheiser hat für den vorliegenden Beitrag nicht relevante Vortragshonorare von Fresenius, Abbott, NOGGO und B.Braun sowie Reisekostenunterstützung von der Firma Deltex Medical erhalten.

Die anderen Autoren erklären, dass keine Interessenkonflikte vorliegen.

\section{Literatur online}

Das Literaturverzeichnis zu diesem Beitrag finden Sie im Internet:

Abonnenten und Nichtabonnenten können unter „www.thieme-connect.de/ ejournals" die Seite der AINS aufrufen und beim jeweiligen Artikel auf „Ergänzendes Material“ klicken - hier ist die Literatur für alle frei zugänglich.

Beitrag online zu finden unter http://dx.doi. org/10.1055/ s-0032-1301375 